\title{
COVID-19 related outcomes among individuals with neurodegenerative diseases: a cohort analysis in the UK biobank
}

Yihan $\mathrm{Hu}^{1,2,3}$, Huazhen Yang ${ }^{1,2}$, Can Hou ${ }^{1,2}$, Wenwen Chen ${ }^{4}$, Hanyue Zhang ${ }^{1,2}$, Zhiye Ying ${ }^{1,2}$, Yao Hu ${ }^{1,2}$, Yajing Sun ${ }^{1,2}$, Yuanyuan Qu ${ }^{1,2}$, Maria Feychting ${ }^{3}$, Unnur Valdimarsdottir ${ }^{5,6}$, Huan Song ${ }^{1,2,5^{*}}$ (D) and Fang Fang ${ }^{3}$

\begin{abstract}
Background: An increased susceptibility to COVID-19 has been suggested for individuals with neurodegenerative diseases, but data are scarce from longitudinal studies.

Methods: In this community-based cohort study, we included 96,275 participants of the UK Biobank who had available SARS-CoV-2 test results in Public Health England. Of these, 2617 had a clinical diagnosis of neurodegenerative diseases in the UK Biobank inpatient hospital data before the outbreak of COVID-19 (defined as January 31st, 2020), while the remaining participants constituted the reference group. We then followed both groups from January 31 st, 2020 to June 14th, 2021 for ascertainment of COVID-19 outcomes, including any COVID-19, inpatient care for COVID19, and COVID-19 related death. Logistic regression was applied to estimate the association between neurogenerative disease and risks of COVID-19 outcomes, adjusted for multiple confounders and somatic comorbidities.

Results: We observed an elevated risk of COVID-19 outcomes among individuals with a neurodegenerative disease compared with the reference group, corresponding to a fully adjusted odds ratio of $2.47(95 \% \mathrm{Cl} 2.25-2.71)$ for any COVID-19, 2.18 (95\%Cl 1.94-2.45) for inpatient COVID-19, and 3.67 (95\%Cl 3.11-4.34) for COVID-19 related death. Among individuals with a positive test result for SARS-CoV-2, individuals with neurodegenerative diseases had also a higher risk of COVID-19 related death than others (fully adjusted odds ratio 2.08; 95\% CI 1.71-2.53).

Conclusion: Among UK Biobank participants who received at least one test for SARS-CoV-2, a pre-existing diagnosis of neurodegenerative disease was associated with a subsequently increased risk of COVID-19, especially COVID-19 related death.
\end{abstract}

Keywords: Neurodegenerative diseases, COVID-19, Cohort study, UK biobank

\section{Background}

The coronavirus disease 2019 (COVID-19), caused by severe acute respiratory syndrome coronavirus 2 (SARS-CoV-2), was declared to be a pandemic by World Health Organization (WHO) in March 2020 [1]. As of

\footnotetext{
*Correspondence: songhuan@wchscu.cn

${ }^{1}$ West China Biomedical Big Data Center and National Clinical Research Center for Geriatrics, West China Hospital, Sichuan University, Guo Xue Lane 37, Chengdu 610041, China

Full list of author information is available at the end of the article
}

September 28th 2021, the disease had led to 232,075,351 confirmed cases and 4,752,988 cases of death. Although most individuals contracted with COVID-19 experience rather mild symptoms [2], elderly people and individuals with chronic diseases are more prone to experience a severe disease course [3-6].

Neurodegenerative diseases are a group of diseases with progressive loss of neurons in the central nervous system. Alzheimer's disease (AD) is the most common neurodegenerative disease, characterized by impairment 
in memory, language, and problems in daily living activities, and is also commonly accompanied with non-cognitive symptoms such as depression [7]. AD affects about $5-7 \%$ of the population aged over 65 years worldwide [8]. With the early death of dopaminergic neurons, Parkinson's disease (PD) mainly affects the motor system, leading to movement disorders typically presented as rest tremor, rigidity, bradykinesia, postural, and gait impairment [9]. Individuals with neurodegenerative diseases are a high-risk population during the COVID-19 pandemic, because of their advanced age and presence of other comorbidities [10-12]. Self-isolation and social distancing due to COVID-19 may have further influence on individuals living with neurodegenerative diseases [13].

To date, only a handful of studies have assessed the association between neurodegenerative diseases and COVID-19, with inconclusive findings [14-22]. This inconsistency may be attributable to different study design, varying sample size and the resulting statistical power, and varying degree of control for important confounders (e.g., comorbidity, lifestyle, and socioeconomic status). We summarized findings of the existing studies in Supplementary Table 1.

To this end, taking advantage of the rich information on lifestyle factors and the complete and timely updated follow-up data for somatic disease and COVID-19 infection in the UK Biobank, we aimed to assess the association between previous neurodegenerative diseases, including primary and vascular neurodegenerative diseases, and subsequent risk of COVID-19.

\section{Methods}

\section{Study design}

UK Biobank is a large prospective study that recruited 502,507 participants aged 40-69years between 2006 and 2010. Baseline data such as demographical factors, lifestyle, and socioeconomic status were collected at recruitment. To ascertain health-related outcomes, the cohort is periodically linked to multiple national datasets [23]. UK Biobank inpatient hospital data include data extracted from Hospital Episode Statistics (HES) from England, Patient Episode Database for Wales (PEDW) from Wales, and the Scottish Morbidity Record from Scotland. Mortality data are derived from multiple death registers, including NHS digital in England and Wales, and NHS central register in Scotland. After the start of COVID-19 outbreak in the UK, COVID-19 test results from Public Health England (PHE), based on real-time RT-PCR (RdRp gene assay) for nose and throat swabs, have also been linked to UK Biobank with updates on a monthly basis.

To reduce the possible bias in COVID-19 detection due to testing capacity, especially in the beginning of the pandemic, in the UK [24], we restricted our analysis to UK Biobank participants who had at least one COVID-19 test record from PHE before June 14th, $2021(n=96,285)$. We further excluded ten participants who were found out to have died before January 31st 2020, leaving 96,275 individuals in the final analyses (Fig. 1). Individuals with an inpatient admission record of any neurodegenerative disease before COVID-19 outbreak (i.e., January 31st, 2020, when the first COVID-19 case was diagnosed in the UK) were included in the exposed group, with the remaining participants included in the unexposed group.

Participation in UK Biobank was voluntary, and all participants gave informed consent before data collection. Ethical approval was received from NHS Research Ethics Committee (REC reference: 16/NW/0274). The present study was approved by the West China Hospital (reference number: 2020.661).

\section{Neurodegenerative diseases}

We identified individuals with neurodegenerative diseases on the basis of UK Biobank inpatient hopital data, according to International Classification of Diseases codes (ICD-10 and ICD-9). The definition of any neurodegenerative disease was a combinition of primary neurodegenerative diseases (ICD-10: F00, F02.0, F02.3, G12.2, G20, G23.1, G23.2, G23.8, G23.9, G25.9, G30, G31.0, G31.1, G31.8, and G31.9, ICD-9: 290.0, 290.1, 331.0, 331.1, 331.2, 331.9, 332.0, 333.0, and 335.2), vascular neurodegenerative diseases (ICD-10: F01, and G21.4, ICD-9: 290.4), and other neurodegenerative diseases (ICD-10: F03, and F05.1, ICD-9: 290.8, and 290.9). In sub-analyses, we also sepcifically analyzed AD (ICD-10: F00, G30, ICD-9: 290.0, 290.1, 331.0) and PD (ICD-10: G20, ICD-9: 332.0). All ICD codes are listed in Supplementary Table 2.

\section{Ascertainment of COVID-19}

By severity, we classified the outcomes of interest as any COVID-19 diagnosis, inpatient COVID-19, and COVID19 related death. Any COVID-19 was defined in case of a positive test result for COVID-19 from PHE, an inpatient diagnosis of COVID-19 from UK Biobank inpatient hospital data, or a death with a cause concerning COVID-19 in the death registers. Inpatient COVID-19 referred to a positive test result from PHE with the origin of inpatient care, or an inpatient diagnosis of COVID-19 based on UK Biobank inpatient hospital data. COVID-19 related death was a death with a cause of death concerning COVID-19 in the death registers. We used ICD-10 codes U07.1 or U07.2 to ascertain inpatient COVID-19 and COVID-19 related deaths (Supplementary Table 2).

In the UK, the initial COVID-19 testing (i.e., before April 27th 2020) was largely restricted to individuals 


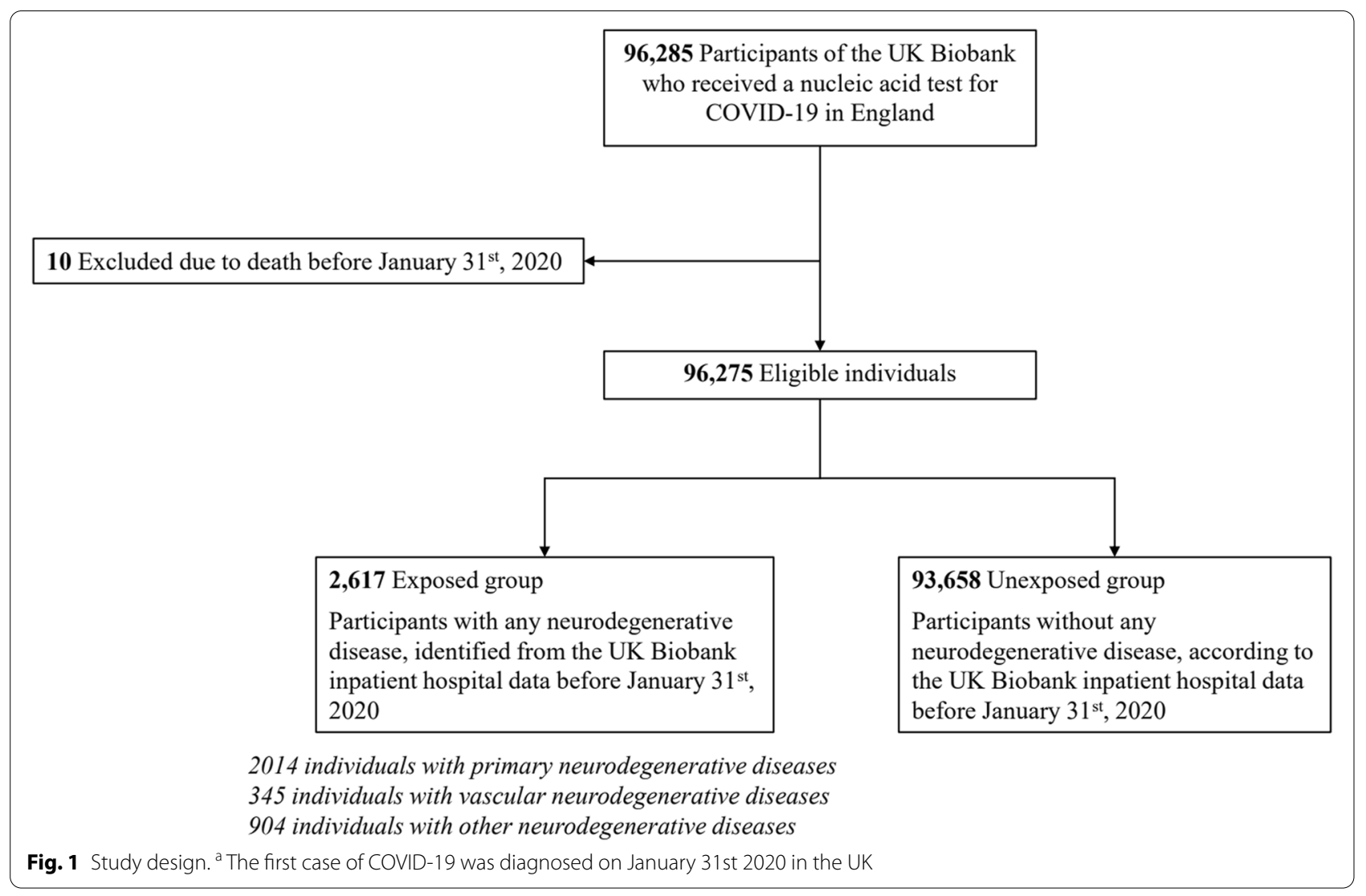

admitted to hospital care and with typical respiratory infection symptoms, which was later extended to other vulnerable populations and community residents because of increased testing capacity [24]. For example, from July 7th 2020 onward, testing has been routinely offered in all care homes in the UK [25].

\section{Covariates}

Covariates such as sociodemographic characteristics (e.g., birth year, sex, and race/ethnicity), socioeconomic status (e.g., educational attainment and annual household income), and lifestyle factors (e.g., smoking and alcohol use) were collected at recruitment through touchscreen questionnaires. Body mass index (BMI) was calculated based on the standing height and weight measurement taken at assessment center. Townsend deprivation score, derived based on the postcode of the participant's registration address, was used to measure material deprivation on a population level, with a greater score representing a greater degree of deprivation [26]. We considered somatic comorbidities as important confounder or mediator for the studied association, and calculated Charlson Comorbidity Index at the time of COVID-19 outbreak for each participant, using the diagnoses of the UK Biobank inpatient hospital data (Supplementary Table 2) [20]. We excluded dementia from this calculation.

\section{Statistical analysis}

The associations between neurodegenerative diseases and different COVID-19 outcomes were assessed by Logistic regression yielding odds ratios (ORs) with 95\% confidence intervals (CIs). All models were adjusted for multiple potential confounders, including birth year (as continuous variable), sex (male or female), race/ethnicity (White, Asian, Black, others, or unknown), the Townsend deprivation index (as a continuous variable), BMI $\left(\leq 24.9 \mathrm{~kg} / \mathrm{m}^{2}, \quad 25.0-29.9 \mathrm{~kg} / \mathrm{m}^{2}, \geq 30.0 \mathrm{~kg} / \mathrm{m}^{2}\right.$, or unknown), annual household income $(<£ 18,000, £ 18,000$ $30,999, £ 31,000-51,999, £ 52,000-100,000,>£ 100,000$, or unknown), educational attainment (college degree, A-level, O-level, Certificate of Secondary Education [CSE] or equivalent, National Vocation Qualifications [NVQ] or equivalent, other professional qualifications, or unknown), smoking (never, ever, or unknown), alcohol use (never, previous, current, or unknown), and Charlson Comorbidity Index (as continuous variable). We analyzed first any neurodegenerative disease and then sub-categories of neurodegenerative diseases (primary, vascular, and 
other neurodegenerative diseases) and specific neurodegenerative diseases (AD and PD).

In subgroup analyses, ORs were calculated by age group ( $\leq 66$ years, $67-75$ years, or $\geq 76$ years, according to tertile distribution), sex (male or female), educational attainment (university degree or below), annual household income $(<£ 18,000, £ 18,000-51,999$, or $\geq £ 52,000)$, BMI $\left(\leq 24.9 \mathrm{~kg} / \mathrm{m}^{2}, \quad 25.0-29.9 \mathrm{~kg} / \mathrm{m}^{2}, \quad\right.$ or $\left.\geq 30.0 \mathrm{~kg} / \mathrm{m}^{2}\right)$, smoking (never or ever), alcohol use (never, previous, or current) and Charlson Comorbidity Index (0, 1, or $\geq 2)$. The difference between sub-group ORs was assessed by introducing interaction terms to the logistic regression or by Wald test.

To explore whether the associations differed by the change of the testing strategy and the dominant variant of the virus in the UK, we did separate analyses for the time periods before April 26th 2020, April 27th 2020 to July 6th 2020, July 7th 2020 to December 16th 2020, and December 17th 2020 onward. Additionally, using the time interval between the first diagnosis of neurodegenerative diseases and cohort entry ( $<1$ year, $1-2$ years, $3-4$ years, or $\geq 5$ years) as a proxy, we assessed the risk of COVID-19 for individuals at different stages of neurodegenerative diseases. Finally, as a spread of COVID-19 was reported in care homes, mainly from March to early May 2020, in the UK [27], which might have affected more individuals with neurodegenerative disease than individuals without such condition, we further studied the risk of COVID-19 related death among all participants with a positive test result of COVID-19. This analysis was aimed to test whether neurodegenerative disease was associated with the susceptibility to COVID-19, especially the severe form, regardless of chances of being infected or tested.

We also performed a few sensitivity analyses to assess the potential influence of residual confounding and selection bias. Because age is the strongest risk factor for both neurodegenerative diseases and severe COVID-19, any residual confounding due to age might lead to an artificial positive association between neurodegenerative diseases and COVID-19. In a sensitivity analysis, we reassessed the studied association using a matched cohort design to further control for age. In this analysis, everyone with neurodegenerative diseases was compared with five individuals without neurodegenerative diseases that were individually matched to the exposed individual by birth year and sex and randomly selected from the study population. Further, although we have restricted the main analysis to UK Biobank participants with at least one COVID-19 test, individuals with neurodegenerative diseases might still differ from individuals without such diseases in terms of access to COVID-19 test (e.g., test through inpatient care in the beginning of the pandemic).
We therefore performed an additional bias analysis, where we assumed 5-100\% underestimate of COVID-19 outcomes among individuals without neurodegenerative diseases and $0 \%$ underestimate among individuals with neurodegenerative diseases. Misclassified cases were randomly selected from individuals without neurodegenerative diseases and were repeatedly selected 100 times for each specific percentage (5-100\%, by every $5 \%$ ). We calculated the mean ORs with 95\% CIs from these repeated analyses and plotted the distribution of the ORs. All the analyses were conducted by Python software (version 3.8) and $\mathrm{R}$ software (version 3.6). A 2 -sided $p<0.05$ was considered statistically significant.

\section{Results}

Among the 96,275 participants included in the analysis, $2617(2.7 \%)$ had a pre-pandemic diagnosis of neurodegenerative disease (exposed group) with the other 93,658 (97.3\%) included in the unexposed group (Fig. 1). The mean age at COVID-19 outbreak was 67.9 years and $46.4 \%$ of the participants were male (Table 1). We observed little difference in race/ethnicity, BMI, smoking, or alcohol use between the exposed and unexposed groups. However, individuals with neurodegenerative disease were older ( 74.2 years vs 67.7 years) and more likely to be male $(53.0 \%$ vs $47.0 \%)$, and had lower socioeconomic status (annual household income $<£ 18,000$ : $34.7 \%$ vs $19.8 \%$ ), lower educational attainment (college or university degree: $20.8 \%$ vs $30.0 \%$ ), greater Townsend deprivation score $(-0.74$ vs -1.20$)$, and higher Charlson Comorbidity Index (2.62 vs 1.29), compared with the unexposed individuals.

As of June 14th, 2021, 17,938 individuals were diagnosed with any COVID-19, including 5749 individuals with inpatient COVID-19 and 1181 with COVID-19 related death. We observed an elevated risk of any COVID-19 (27.4\% vs 18.4\%), inpatient COVID19 (14.5\% vs $5.7 \%)$, and COVID-19 related death (7.5\% vs $1.1 \%)$ among individuals with neurodegenerative disease compared with the unexposed individuals (Table 1). This corresponds to a fully adjusted OR of 2.47 (95\%CI 2.25-2.71) for any COVID-19, 2.18 (95\%CI 1.94-2.45) for inpatient COVID-19, and 3.67 (95\%CI 3.11-4.34) for COVID-19 related death, after adjusting for all available confounders (Fig. 2).

In the analyses for subtypes of neurodegenerative diseases, the elevated risk of any COVID-19 was more pronounced for vascular neurodegenerative diseases, compared with primary neurodegenerative diseases (OR, 4.79 [95\%CI 3.84-5.97] vs 2.17 [95\%CI $1.95-$ 2.41], $p$ value for difference<0.001) (Fig. 2). No difference was noted for inpatient COVID-19 or COVID-19 related death. We found an increased risk of all studied 
Table 1 Characteristics of the study cohort

\begin{tabular}{|c|c|c|c|}
\hline Characteristics & $\begin{array}{l}\text { Individuals with neurodegenerative } \\
\text { diseases }(n=2617)\end{array}$ & $\begin{array}{l}\text { Individuals without } \\
\text { neurodegenerative diseases } \\
(n=93,658)\end{array}$ & Total $(n=96,275)$ \\
\hline Mean age (SD) at outbreak ${ }^{\mathrm{a}}$, years & $74.2(5.84)$ & $67.7(8.23)$ & $67.9(8.25)$ \\
\hline \multicolumn{4}{|l|}{ Age group, years } \\
\hline$\leq 66$ & $187(7.1)$ & $30,254(32.3)$ & $30,441(31.6)$ \\
\hline $67-75$ & $436(16.7)$ & $26,367(28.2)$ & $26,803(27.8)$ \\
\hline$\geq 76$ & $1994(76.2)$ & $37,037(39.5)$ & $39,031(40.5)$ \\
\hline \multicolumn{4}{|l|}{ Sex } \\
\hline Female & $1230(47.0)$ & $50,369(53.8)$ & $51,599(53.6)$ \\
\hline Male & $1387(53.0)$ & $43,289(46.2)$ & $44,676(46.4)$ \\
\hline \multicolumn{4}{|l|}{ Race/ethnicity } \\
\hline White & $2407(92.0)$ & $83,989(89.7)$ & $86,396(89.7)$ \\
\hline Other & $189(7.2)$ & $9136(9.8)$ & $9325(9.7)$ \\
\hline Unknown & $21(0.8)$ & $533(0.6)$ & $554(0.6)$ \\
\hline \multicolumn{4}{|l|}{ Body mass index, $\mathrm{kg} / \mathrm{m}^{2}$} \\
\hline$<24.9$ & $696(26.6)$ & $27,367(29.2)$ & $28,063(29.1)$ \\
\hline $24.9-29.9$ & $1082(41.3)$ & $39,782(42.5)$ & $40,864(42.4)$ \\
\hline$\geq 30$ & $797(30.5)$ & $25,885(27.6)$ & $26,682(27.7)$ \\
\hline Unknown & $42(1.6)$ & $624(0.7)$ & $666(0.7)$ \\
\hline \multicolumn{4}{|l|}{ Smoking } \\
\hline Never & $1287(49.2)$ & $48,823(52.1)$ & $50,110(52.0)$ \\
\hline Ever & $1296(49.5)$ & $44,200(47.2)$ & $45,496(47.3)$ \\
\hline Unknown & $34(1.3)$ & $635(0.7)$ & $669(0.7)$ \\
\hline \multicolumn{4}{|l|}{ Alcohol use } \\
\hline Never & $176(6.7)$ & $4420(4.7)$ & $4596(4.8)$ \\
\hline Previous & $182(7.0)$ & $3534(3.8)$ & $3716(3.9)$ \\
\hline Current & $2237(85.5)$ & $85,364(91.1)$ & $87,601(91.0)$ \\
\hline Unknown & $22(0.8)$ & $340(0.4)$ & $362(0.4)$ \\
\hline Townsend deprivation index & $-0.74(3.41)$ & $-1.20(3.12)$ & $-1.19(3.13)$ \\
\hline \multicolumn{4}{|l|}{ Annual household income, $£$} \\
\hline$<18,000$ & $909(34.7)$ & $18,534(19.8)$ & $19,443(20.2)$ \\
\hline $18,000-30,999$ & $556(21.2)$ & $19,911(21.3)$ & $20,467(21.3)$ \\
\hline $31,000-51,999$ & $290(11.1)$ & $20,358(21.7)$ & $20,648(21.4)$ \\
\hline $52,000-100,000$ & $168(6.4)$ & $15,601(16.7)$ & $15,769(16.4)$ \\
\hline$>100,000$ & $50(1.9)$ & $4577(4.9)$ & $4627(4.8)$ \\
\hline Unknown & $644(24.6)$ & $14,677(15.7)$ & $15,321(15.9)$ \\
\hline \multicolumn{4}{|l|}{ Educational attainment } \\
\hline College or University degree & $544(20.8)$ & $28,109(30.0)$ & $28,653(29.8)$ \\
\hline A levels/AS levels or equivalent & $198(7.6)$ & $9953(10.6)$ & $10,151(10.5)$ \\
\hline O levels/GCSEs or equivalent & $491(18.8)$ & $19,969(21.3)$ & $20,460(21.3)$ \\
\hline CSEs or equivalent & $72(2.8)$ & $5545(5.9)$ & $5617(5.8)$ \\
\hline NVQ or HND or HNC or equivalent & $187(7.1)$ & $6550(7.0)$ & $6737(7.0)$ \\
\hline Other professional qualifications & $158(6.0)$ & $4964(5.3)$ & $5122(5.3)$ \\
\hline Unknown & $967(37.0)$ & $18,568(19.8)$ & $19,535(20.3)$ \\
\hline Charlson Comorbidity Index & $2.62(2.78)$ & $1.29(2.16)$ & $1.32(2.19)$ \\
\hline \multicolumn{4}{|l|}{ Any COVID-19 } \\
\hline Yes & $718(27.4)$ & $17,220(18.4)$ & $17,938(18.6)$ \\
\hline No & $1899(72.6)$ & $76,438(81.6)$ & $78,337(81.4)$ \\
\hline \multicolumn{4}{|l|}{ Inpatient COVID-19 } \\
\hline Yes & $379(14.5)$ & $5370(5.7)$ & $5749(6.0)$ \\
\hline
\end{tabular}


Table 1 (continued)

\begin{tabular}{llll}
\hline Characteristics & $\begin{array}{l}\text { Individuals with neurodegenerative } \\
\text { diseases }(\boldsymbol{n = 2 6 1 7 )}\end{array}$ & $\begin{array}{l}\text { Individuals without } \\
\text { neurodegenerative diseases } \\
(\boldsymbol{n}=\mathbf{9 3 , 6 5 8 )}\end{array}$ & Total $(\boldsymbol{n}=\mathbf{9 6 , 2 7 5 )}$ \\
\hline $\begin{array}{l}\text { No } \\
\text { COVID-19 related death }\end{array}$ & $2238(85.5)$ & $88,288(94.3)$ & $90,526(94.0)$ \\
Yes & $195(7.5)$ & $986(1.1)$ & $1181(1.2)$ \\
No & $2422(92.5)$ & $92,672(98.9)$ & $95,094(98.8)$ \\
\hline
\end{tabular}

Data are $\mathrm{n} / \mathrm{N}(\%)$, unless otherwise specified

${ }^{\text {a }}$ The first case of COVID-19 was diagnosed on January 31st 2020 in the UK

Any COVID-19 includes a positive test result of COVID-19 according to Public Health England, an inpatient diagnosis from UK Biobank inpatient hospital data, or a death related to COVID-19

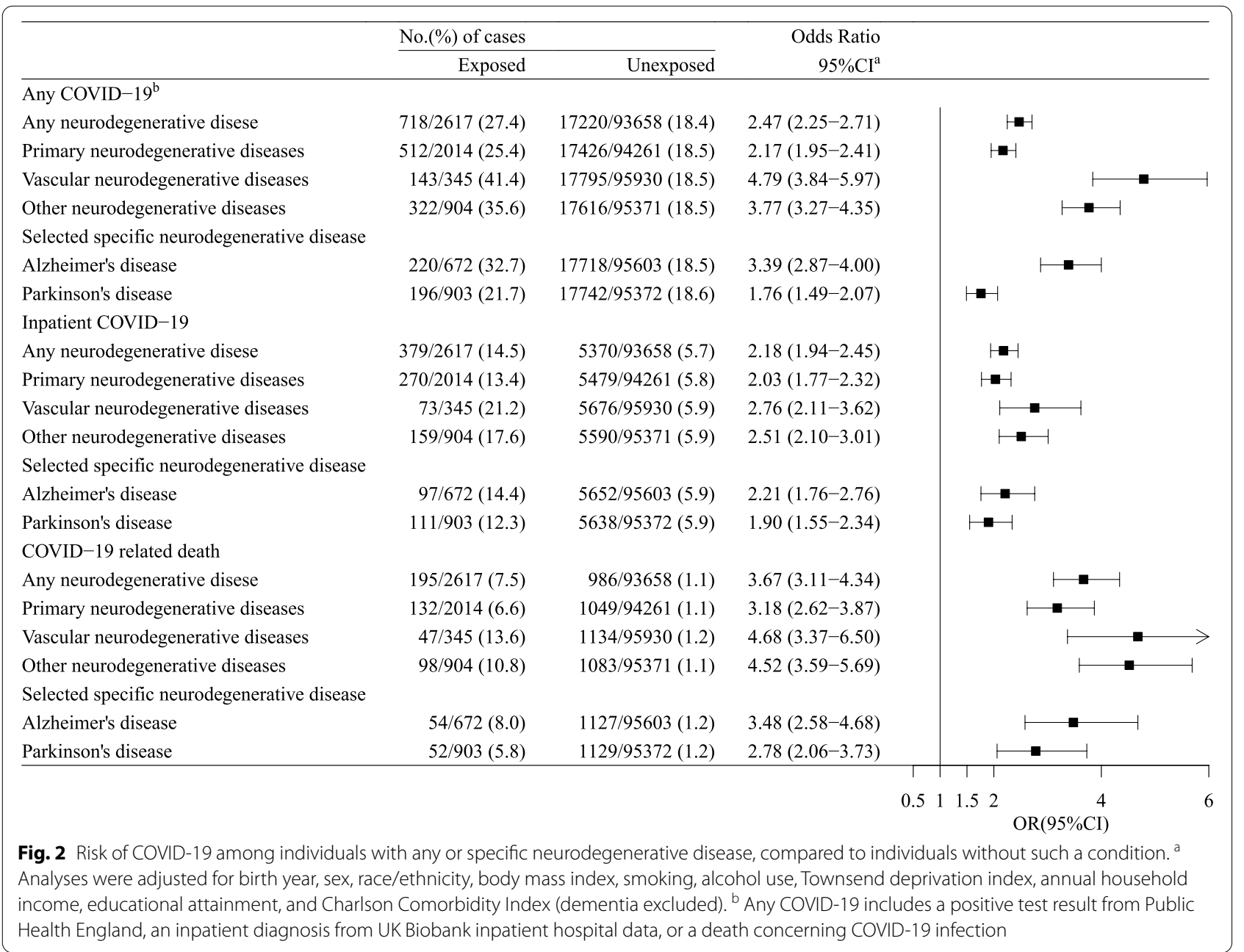

COVID-19 outcomes among individuals with PD as well as individuals with AD (Fig. 2).

Similar associations were observed across different groups of age, sex, BMI, smoking, alcohol use, annual household income, educational attainment, and Charlson Comorbidity Index (Table 2). A positive association for any COVID-19 was noted during all time periods (OR 1.49 [95\%CI 1.13-1.97] before April 26th 2020, OR 4.00 [95\%CI 3.11-5.15] during April 27th 2020-July 6th 2020, OR 1.72 [95\%CI 1.47-2.01] during July 7th 2020 -December 16th 2020, and OR 2.52 [95\%CI 2.15-2.96] from December 17th 2020 onward) (Table 2). No clear trend 
Table 2 Risk of COVID-19 among individuals with neurodegenerative diseases, compared to individuals without such condition, an analysis of UK Biobank data

\begin{tabular}{|c|c|c|c|c|c|c|c|c|c|}
\hline \multirow[t]{2}{*}{ Characteristics } & \multicolumn{2}{|c|}{$\begin{array}{l}\text { No.(\%)of any COVID-19 } \\
\text { cases }\end{array}$} & \multirow{2}{*}{$\begin{array}{l}\text { Odds Ratio } \\
(95 \% \mathrm{Cl})\end{array}$} & \multicolumn{2}{|c|}{$\begin{array}{l}\text { No.(\%)of Inpatient } \\
\text { COVID-19 }\end{array}$} & \multirow{2}{*}{$\begin{array}{l}\text { Odds Ratio } \\
(95 \% \mathrm{Cl})\end{array}$} & \multicolumn{2}{|c|}{$\begin{array}{l}\text { No.(\%)of COVID-19 } \\
\text { related death }\end{array}$} & \multirow{2}{*}{$\begin{array}{l}\text { Odds Ratio } \\
(95 \% \mathrm{Cl})\end{array}$} \\
\hline & Exposed & Unexposed & & Exposed & Unexposed & & Exposed & Unexposed & \\
\hline \multicolumn{10}{|l|}{ Sex } \\
\hline Female & $\begin{array}{l}347 / 1230 \\
(28.2)\end{array}$ & $\begin{array}{l}9082 / 50369 \\
(18.0)\end{array}$ & $\begin{array}{l}2.81 \\
(2.46-3.21)\end{array}$ & $\begin{array}{l}164 / 1230 \\
(13.3)\end{array}$ & $\begin{array}{l}2519 / 50369 \\
(5.0)\end{array}$ & $\begin{array}{l}2.46 \\
(2.06-2.93)\end{array}$ & $\begin{array}{l}76 / 1230 \\
(6.2)\end{array}$ & $\begin{array}{l}355 / 50369 \\
(0.7)\end{array}$ & $\begin{array}{l}4.27 \\
(3.26-5.59)\end{array}$ \\
\hline Male & $\begin{array}{l}371 / 1387 \\
(26.7)\end{array}$ & $\begin{array}{l}8138 / 43289 \\
(18.8)\end{array}$ & $\begin{array}{l}2.20 \\
(1.94-2.50)\end{array}$ & $\begin{array}{l}215 / 1387 \\
(15.5)\end{array}$ & $\begin{array}{l}2851 / 43289 \\
(6.6)\end{array}$ & $\begin{array}{l}1.99 \\
(1.70-2.33)\end{array}$ & $\begin{array}{l}119 / 1387 \\
(8.6)\end{array}$ & $\begin{array}{l}631 / 43289 \\
(1.5)\end{array}$ & $\begin{array}{l}3.37 \\
(2.72-4.17)\end{array}$ \\
\hline \multicolumn{10}{|l|}{ Age, years } \\
\hline$\leq 66$ & $50 / 187(26.7)$ & $\begin{array}{l}8736 / 30254 \\
(28.9)\end{array}$ & $\begin{array}{l}1.05 \\
(0.76-1.47)\end{array}$ & 28/187 (15.0) & $\begin{array}{l}1776 / 30254 \\
(5.9)\end{array}$ & $\begin{array}{l}2.06 \\
(1.36-3.14)\end{array}$ & $7 / 187(3.7)$ & $\begin{array}{l}87 / 30254 \\
(0.3)\end{array}$ & $\begin{array}{l}4.01 \\
(1.63-9.89)\end{array}$ \\
\hline $67-75$ & $95 / 436(21.8)$ & $\begin{array}{l}4046 / 26367 \\
(15.3)\end{array}$ & $\begin{array}{l}1.44 \\
(1.14-1.82)\end{array}$ & $47 / 436(10.8)$ & $\begin{array}{l}1287 / 26367 \\
(4.9)\end{array}$ & $\begin{array}{l}1.59 \\
(1.15-2.19)\end{array}$ & 20/436 (4.6) & $\begin{array}{l}219 / 26367 \\
(0.8)\end{array}$ & $\begin{array}{l}3.13 \\
(1.91-5.15)\end{array}$ \\
\hline$\geq 76$ & $\begin{array}{l}573 / 1994 \\
(28.7)\end{array}$ & $\begin{array}{l}4438 / 37037 \\
(12.0)\end{array}$ & $\begin{array}{l}2.73 \\
(2.46-3.03)\end{array}$ & $\begin{array}{l}304 / 1994 \\
(15.2)\end{array}$ & $\begin{array}{l}2307 / 37037 \\
(6.2)\end{array}$ & $\begin{array}{l}2.25 \\
(1.97-2.57)\end{array}$ & $\begin{array}{l}168 / 1994 \\
(8.4)\end{array}$ & $\begin{array}{l}680 / 37037 \\
(1.8)\end{array}$ & $\begin{array}{l}4.03 \\
(3.36-4.83)\end{array}$ \\
\hline \multicolumn{10}{|l|}{$\mathrm{BMI}, \mathrm{kg} / \mathrm{m}^{2}$} \\
\hline$<24.9$ & $\begin{array}{l}182 / 696 \\
(26.1)\end{array}$ & $\begin{array}{l}4424 / 27367 \\
(16.2)\end{array}$ & $\begin{array}{l}3.09 \\
(2.58-3.71)\end{array}$ & $91 / 696(13.1)$ & $\begin{array}{l}1029 / 27367 \\
(3.8)\end{array}$ & $\begin{array}{l}3.14 \\
(2.47-3.99)\end{array}$ & $52 / 696(7.5)$ & $\begin{array}{l}130 / 27367 \\
(0.5)\end{array}$ & $\begin{array}{l}7.41 \\
(5.21-10.54)\end{array}$ \\
\hline $25.0-29.9$ & $\begin{array}{l}317 / 1082 \\
(29.3)\end{array}$ & $\begin{array}{l}7203 / 39782 \\
(18.1)\end{array}$ & $\begin{array}{l}2.85 \\
(2.48-3.27)\end{array}$ & $\begin{array}{l}161 / 1082 \\
(14.9)\end{array}$ & $\begin{array}{l}2155 / 39782 \\
(5.4)\end{array}$ & $\begin{array}{l}2.46 \\
(2.06-2.95)\end{array}$ & $\begin{array}{l}90 / 1082 \\
(8.3)\end{array}$ & $\begin{array}{l}372 / 39782 \\
(0.9)\end{array}$ & $\begin{array}{l}4.91 \\
(3.82-6.31)\end{array}$ \\
\hline$\geq 30$ & $\begin{array}{l}208 / 797 \\
(26.1)\end{array}$ & $\begin{array}{l}5447 / 25885 \\
(21.0)\end{array}$ & $\begin{array}{l}1.77 \\
(1.50-2.09)\end{array}$ & $\begin{array}{l}120 / 797 \\
(15.1)\end{array}$ & $\begin{array}{l}2120 / 25885 \\
(8.2)\end{array}$ & $\begin{array}{l}1.57 \\
(1.28-1.93)\end{array}$ & $50 / 797(6.3)$ & $\begin{array}{l}462 / 25885 \\
(1.8)\end{array}$ & $\begin{array}{l}1.88 \\
(1.37-2.57)\end{array}$ \\
\hline \multicolumn{10}{|l|}{ Smoking } \\
\hline Never & $\begin{array}{l}325 / 1287 \\
(25.3)\end{array}$ & $\begin{array}{l}9076 / 48823 \\
(18.6)\end{array}$ & $\begin{array}{l}2.43 \\
(2.12-2.78)\end{array}$ & $\begin{array}{l}162 / 1287 \\
(12.6)\end{array}$ & $\begin{array}{l}2460 / 48823 \\
(5.0)\end{array}$ & $\begin{array}{l}2.33 \\
(1.95-2.78)\end{array}$ & $\begin{array}{l}79 / 1287 \\
(6.1)\end{array}$ & $\begin{array}{l}358 / 48823 \\
(0.7)\end{array}$ & $\begin{array}{l}4.05 \\
(3.11-5.27)\end{array}$ \\
\hline Ever & $\begin{array}{l}381 / 1296 \\
(29.4)\end{array}$ & $\begin{array}{l}8033 / 44200 \\
(18.2)\end{array}$ & $\begin{array}{l}2.51 \\
(2.21-2.85)\end{array}$ & $\begin{array}{l}211 / 1296 \\
(16.3)\end{array}$ & $\begin{array}{l}2857 / 44200 \\
(6.5)\end{array}$ & $\begin{array}{l}2.09 \\
(1.78-2.45)\end{array}$ & $\begin{array}{l}115 / 1296 \\
(8.9)\end{array}$ & $\begin{array}{l}613 / 44200 \\
(1.4)\end{array}$ & $\begin{array}{l}3.57 \\
(2.87-4.44)\end{array}$ \\
\hline \multicolumn{10}{|l|}{ Alcohol use } \\
\hline Never & $48 / 176(27.3)$ & $\begin{array}{l}1072 / 4420 \\
(24.3)\end{array}$ & $\begin{array}{l}1.75 \\
(1.22-2.51)\end{array}$ & $30 / 176(17.0)$ & $\begin{array}{l}381 / 4420 \\
(8.6)\end{array}$ & $\begin{array}{l}1.81 \\
(1.18-2.78)\end{array}$ & 6/176 (3.4) & $\begin{array}{l}79 / 4420 \\
(1.8)\end{array}$ & $\begin{array}{l}0.99 \\
(0.41-2.36)\end{array}$ \\
\hline Previous & 63/182 (34.6) & $\begin{array}{l}693 / 3534 \\
(19.6)\end{array}$ & $\begin{array}{l}2.34 \\
(1.68-3.24)\end{array}$ & $41 / 182(22.5)$ & $\begin{array}{l}315 / 3534 \\
(8.9)\end{array}$ & $\begin{array}{l}2.13 \\
(1.45-3.14)\end{array}$ & $\begin{array}{l}20 / 182 \\
(11.0)\end{array}$ & $\begin{array}{l}72 / 3534 \\
(2.0)\end{array}$ & $\begin{array}{l}3.58 \\
(2.05-6.24)\end{array}$ \\
\hline Current & $\begin{array}{l}602 / 2237 \\
(26.9)\end{array}$ & $\begin{array}{l}15,385 / 85364 \\
(18.0)\end{array}$ & $\begin{array}{l}2.55 \\
(2.31-2.82)\end{array}$ & $\begin{array}{l}305 / 2237 \\
(13.6)\end{array}$ & $\begin{array}{l}4643 / 85364 \\
(5.4)\end{array}$ & $\begin{array}{l}2.23 \\
(1.96-2.54)\end{array}$ & $\begin{array}{l}168 / 2237 \\
(7.5)\end{array}$ & $\begin{array}{l}824 / 85364 \\
(1.0)\end{array}$ & $\begin{array}{l}4.15 \\
(3.47-4.98)\end{array}$ \\
\hline \multicolumn{10}{|c|}{ Annual household income, $£$} \\
\hline$<18,000$ & $\begin{array}{l}279 / 909 \\
(30.7)\end{array}$ & $\begin{array}{l}3501 / 18534 \\
(18.9)\end{array}$ & $\begin{array}{l}2.44 \\
(2.10-2.84)\end{array}$ & $\begin{array}{l}158 / 909 \\
(17.4)\end{array}$ & $\begin{array}{l}1490 / 18534 \\
(8.0)\end{array}$ & $\begin{array}{l}2.11 \\
(1.75-2.54)\end{array}$ & $75 / 909(8.3)$ & $\begin{array}{l}368 / 18534 \\
(2.0)\end{array}$ & $\begin{array}{l}3.05 \\
(2.33-3.99)\end{array}$ \\
\hline $18,000-51,999$ & $\begin{array}{l}197 / 846 \\
(23.3)\end{array}$ & $\begin{array}{l}7597 / 40269 \\
(18.9)\end{array}$ & $\begin{array}{l}2.28 \\
(1.93-2.70)\end{array}$ & $94 / 846(11.1)$ & $\begin{array}{l}2120 / 40269 \\
(5.3)\end{array}$ & $\begin{array}{l}2.21 \\
(1.77-2.77)\end{array}$ & $52 / 846(6.1)$ & $\begin{array}{l}335 / 40269 \\
(0.8)\end{array}$ & $\begin{array}{l}4.44 \\
(3.25-6.07)\end{array}$ \\
\hline$>52,000$ & 45/218 (20.6) & $\begin{array}{l}3560 / 20178 \\
(17.6)\end{array}$ & $\begin{array}{l}2.13 \\
(1.52-3.00)\end{array}$ & 15/218 (6.9) & $\begin{array}{l}761 / 20178 \\
(3.8)\end{array}$ & $\begin{array}{l}1.84 \\
(1.07-3.16)\end{array}$ & $15 / 218(6.9)$ & $\begin{array}{l}81 / 20178 \\
(0.4)\end{array}$ & $\begin{array}{l}8.61 \\
(4.69-15.78)\end{array}$ \\
\hline \multicolumn{10}{|c|}{ Educational attainment } \\
\hline $\begin{array}{l}\text { University } \\
\text { degree }\end{array}$ & $\begin{array}{l}115 / 544 \\
(21.1)\end{array}$ & $\begin{array}{l}4307 / 28109 \\
(15.3)\end{array}$ & $\begin{array}{l}2.38 \\
(1.92-2.96)\end{array}$ & $66 / 544(12.1)$ & $\begin{array}{l}1170 / 28109 \\
(4.2)\end{array}$ & $\begin{array}{l}2.76 \\
(2.10-3.64)\end{array}$ & $32 / 544(5.9)$ & $\begin{array}{l}186 / 28109 \\
(0.7)\end{array}$ & $\begin{array}{l}4.70 \\
(3.14-7.04)\end{array}$ \\
\hline $\begin{array}{l}\text { Without Uni- } \\
\text { versity degree }\end{array}$ & $\begin{array}{l}290 / 1106 \\
(26.2)\end{array}$ & $\begin{array}{l}9310 / 46981 \\
(19.8)\end{array}$ & $\begin{array}{l}2.45 \\
(2.13-2.83)\end{array}$ & $\begin{array}{l}139 / 1106 \\
(12.6)\end{array}$ & $\begin{array}{l}2639 / 46981 \\
(5.6)\end{array}$ & $\begin{array}{l}2.21 \\
(1.83-2.67)\end{array}$ & $\begin{array}{l}81 / 1106 \\
(7.3)\end{array}$ & $\begin{array}{l}413 / 46981 \\
(0.9)\end{array}$ & $\begin{array}{l}4.59 \\
(3.55-5.95)\end{array}$ \\
\hline \multicolumn{10}{|c|}{ Charlson Comorbidity Index } \\
\hline 0 & $\begin{array}{l}184 / 678 \\
(27.1)\end{array}$ & $\begin{array}{l}10,422 / 51302 \\
(20.3)\end{array}$ & $\begin{array}{l}2.59 \\
(2.17-3.09)\end{array}$ & $80 / 678(11.8)$ & $\begin{array}{l}2264 / 51302 \\
(4.4)\end{array}$ & $\begin{array}{l}3.17 \\
(2.49-4.05)\end{array}$ & $46 / 678(6.8)$ & $\begin{array}{l}238 / 51302 \\
(0.5)\end{array}$ & $\begin{array}{l}8.21 \\
(5.82-11.57)\end{array}$ \\
\hline 1 & $\begin{array}{l}122 / 485 \\
(25.2)\end{array}$ & $\begin{array}{l}2760 / 15181 \\
(18.2)\end{array}$ & $\begin{array}{l}2.21 \\
(1.78-2.75)\end{array}$ & $59 / 485(12.2)$ & $\begin{array}{l}933 / 15181 \\
(6.1)\end{array}$ & $\begin{array}{l}2.22 \\
(1.67-2.96)\end{array}$ & $31 / 485(6.4)$ & $\begin{array}{l}157 / 15181 \\
(1.0)\end{array}$ & $\begin{array}{l}4.81 \\
(3.18-7.28)\end{array}$ \\
\hline$\geq 2$ & $\begin{array}{l}412 / 1454 \\
(28.3)\end{array}$ & $\begin{array}{l}4038 / 27175 \\
(14.9)\end{array}$ & $\begin{array}{l}2.33 \\
(2.06-2.64)\end{array}$ & $\begin{array}{l}240 / 1454 \\
(16.5)\end{array}$ & $\begin{array}{l}2173 / 27175 \\
(8.0)\end{array}$ & $\begin{array}{l}1.90 \\
(1.64-2.21)\end{array}$ & $\begin{array}{l}118 / 1454 \\
(8.1)\end{array}$ & $\begin{array}{l}591 / 27175 \\
(2.2)\end{array}$ & $\begin{array}{l}2.85 \\
(2.30-3.52)\end{array}$ \\
\hline
\end{tabular}


Table 2 (continued)

\begin{tabular}{|c|c|c|c|c|c|c|c|c|c|}
\hline \multirow[t]{2}{*}{ Characteristics } & \multicolumn{2}{|c|}{$\begin{array}{l}\text { No.(\%)of any COVID-19 } \\
\text { cases }\end{array}$} & \multirow{2}{*}{$\begin{array}{l}\text { Odds Ratio } \\
(95 \% \mathrm{Cl})\end{array}$} & \multicolumn{2}{|c|}{$\begin{array}{l}\text { No.(\%)of Inpatient } \\
\text { COVID-19 }\end{array}$} & \multirow{2}{*}{$\begin{array}{l}\text { Odds Ratio } \\
(95 \% \mathrm{Cl})\end{array}$} & \multicolumn{2}{|c|}{$\begin{array}{l}\text { No.(\%)of COVID-19 } \\
\text { related death }\end{array}$} & \multirow{2}{*}{$\begin{array}{l}\text { Odds Ratio } \\
(95 \% \mathrm{Cl})\end{array}$} \\
\hline & Exposed & Unexposed & & Exposed & Unexposed & & Exposed & Unexposed & \\
\hline \multicolumn{10}{|l|}{ Calendar period } \\
\hline $\begin{array}{l}\text { Before April } \\
\text { 26th } 2020\end{array}$ & $\begin{array}{l}162 / 258 \\
(62.8)\end{array}$ & $\begin{array}{l}1218 / 2353 \\
(51.8)\end{array}$ & $\begin{array}{l}1.49 \\
(1.13-1.97)\end{array}$ & $\begin{array}{l}106 / 258 \\
(41.1)\end{array}$ & $\begin{array}{l}1030 / 2353 \\
(43.8)\end{array}$ & $\begin{array}{l}0.77 \\
(0.59-1.02)\end{array}$ & $\begin{array}{l}87 / 258 \\
(33.7)\end{array}$ & $\begin{array}{l}295 / 2353 \\
(12.5)\end{array}$ & $\begin{array}{l}2.19 \\
(1.61-2.97)\end{array}$ \\
\hline $\begin{array}{l}\text { April 27th } \\
2020 \text { to July 6th } \\
2020\end{array}$ & $\begin{array}{l}101 / 427 \\
(23.7)\end{array}$ & $630 / 7456(8.4)$ & $\begin{array}{l}4.00 \\
(3.11-5.15)\end{array}$ & 47/427 (11.0) & $\begin{array}{l}325 / 7456 \\
(4.4)\end{array}$ & $\begin{array}{l}2.42 \\
(1.73-3.39)\end{array}$ & 20/427 (4.7) & $\begin{array}{l}58 / 7456 \\
(0.8)\end{array}$ & $\begin{array}{l}4.02 \\
(2.33-6.93)\end{array}$ \\
\hline $\begin{array}{l}\text { July 7th } 2020 \\
\text { to Dec } 16 \text { th } \\
2020\end{array}$ & $\begin{array}{l}226 / 1040 \\
(21.7)\end{array}$ & $\begin{array}{l}7060 / 35252 \\
(20.0)\end{array}$ & $\begin{array}{l}1.72 \\
(1.47-2.01)\end{array}$ & $\begin{array}{l}117 / 1040 \\
(11.2)\end{array}$ & $\begin{array}{l}1874 / 35252 \\
(5.3)\end{array}$ & $\begin{array}{l}1.85 \\
(1.50-2.27)\end{array}$ & $\begin{array}{l}41 / 1040 \\
(3.9)\end{array}$ & $\begin{array}{l}277 / 35252 \\
(0.8)\end{array}$ & $\begin{array}{l}2.46 \\
(1.74-3.49)\end{array}$ \\
\hline $\begin{array}{l}\text { Dec 17th } 2020 \\
\text { onward }\end{array}$ & $\begin{array}{l}229 / 892 \\
(25.7)\end{array}$ & $\begin{array}{l}8312 / 48597 \\
(17.1)\end{array}$ & $\begin{array}{l}2.52 \\
(2.15-2.96)\end{array}$ & $\begin{array}{l}109 / 892 \\
(12.2)\end{array}$ & $\begin{array}{l}2141 / 48597 \\
(4.4)\end{array}$ & $\begin{array}{l}2.27 \\
(1.83-2.82)\end{array}$ & 47/892 (5.3) & $\begin{array}{l}356 / 48597 \\
(0.7)\end{array}$ & $\begin{array}{l}3.42 \\
(2.47-4.75)\end{array}$ \\
\hline
\end{tabular}

Odds Ratio (95\%) were derived from logistic regression models, which were adjusted for birth year, sex, race/ethnicity, body mass index, smoking, alcohol use, Townsend deprivation index, annual household income, educational attainment, and Charlson Comorbidity Index

was observed for time since the first diagnosis of neurodegenerative diseases (Table 3). Finally, among participants with a positive test result for COVID-19, there was also an increased risk of COVID-19 related death among individual with neurodegenerative diseases than others (OR 2.08; 95\%CI 1.71-2.53) (Table 4).

In the sensitivity analysis using a matched cohort design, including 2617 exposed individuals and 11,571 birth year- and sex- matched unexposed individuals, we obtained largely comparable results (Supplementary Fig. 1) as in the main analysis. The fully adjusted OR was 2.17 (95\%CI 1.96-2.41) for any COVID-19, 2.17 (95\%CI 1.89-2.48) for inpatient COVID-19, and 3.90 (95\%CI 3.17-4.79) for COVID-19 related death. In the bias analysis, we found a positive association between neurodegenerative disease and all COVID-19 outcomes when assuming different degrees of underestimation of
COVID-19 outcomes among individuals without neurodegenerative diseases, although the association was not statistically significant for any COVID-19 when assuming $>65 \%$ underestimation among individuals without neurodegenerative diseases (Supplementary Fig. 2).

\section{Discussion}

To the best of our knowledge, this is the first cohort study to comprehensively assess the association between multiple neurodegenerative diseases and the risk of COVID-19 related outcomes. Among the UK Biobank participants who received at least one COVID-19 test, we found individuals with a previous neurodegenerative disease to be at an elevated risk of COVID-19, including any COVID-19 infection, hospitalization for COVID-19, and death due to COVID-19. The association was independent from multiple confounders, such as age and somatic

Table 3 Risk of COVID-19 among individuals with neurodegenerative diseases, by time since the first diagnosis, compared to individuals without such conditions

\begin{tabular}{|c|c|c|c|c|c|c|c|c|c|}
\hline & \multicolumn{2}{|c|}{ No.(\%)of Any COVID-19 } & \multirow{2}{*}{$\begin{array}{l}\text { Odds Ratio } \\
(95 \% \mathrm{Cl})\end{array}$} & \multicolumn{2}{|c|}{ No.(\%)of Inpatient COVID-19 } & \multirow{2}{*}{$\begin{array}{l}\text { Odds Ratio } \\
(95 \% \mathrm{Cl})\end{array}$} & \multicolumn{2}{|c|}{$\begin{array}{l}\text { No.(\%)of COVID-19 related } \\
\text { death }\end{array}$} & \multirow{2}{*}{$\begin{array}{l}\text { Odds Ratio } \\
(95 \% \mathrm{Cl})\end{array}$} \\
\hline & Exposed & Unexposed & & Exposed & Unexposed & & Exposed & Unexposed & \\
\hline \multicolumn{10}{|c|}{ Time since the first diagnosis of neurodegenerative diseases, years } \\
\hline$<1$ & $82 / 309(26.5)$ & $\begin{array}{l}17,220 / 93658 \\
(18.4)\end{array}$ & $\begin{array}{l}2.56 \\
(1.97-3.32)\end{array}$ & $49 / 309(15.9)$ & $\begin{array}{l}5370 / 93658 \\
(5.7)\end{array}$ & $\begin{array}{l}2.45 \\
(1.79-3.36)\end{array}$ & $19 / 309(6.1)$ & $\begin{array}{l}986 / 93658 \\
(1.1)\end{array}$ & $\begin{array}{l}2.81 \\
(1.73-4.57)\end{array}$ \\
\hline $1-2$ & $\begin{array}{l}289 / 1009 \\
(28.6)\end{array}$ & & $\begin{array}{l}2.70 \\
(2.34-3.12)\end{array}$ & $\begin{array}{l}158 / 1009 \\
(15.7)\end{array}$ & & $\begin{array}{l}2.37 \\
(1.98-2.83)\end{array}$ & $84 / 1009(8.3)$ & & $\begin{array}{l}3.92 \\
(3.08-5.01)\end{array}$ \\
\hline $3-4$ & $145 / 549(26.4)$ & & $\begin{array}{l}2.42 \\
(1.99-2.95)\end{array}$ & $72 / 549(13.1)$ & & $\begin{array}{l}1.95 \\
(1.51-2.51)\end{array}$ & $49 / 549(8.9)$ & & $\begin{array}{l}4.45 \\
(3.25-6.09)\end{array}$ \\
\hline$\geq 5$ & $202 / 750(26.9)$ & & $\begin{array}{l}2.27 \\
(1.92-2.68)\end{array}$ & $100 / 750(13.3)$ & & $\begin{array}{l}1.99 \\
(1.60-2.47)\end{array}$ & $43 / 750(5.7)$ & & $\begin{array}{l}2.89 \\
(2.08-4.00)\end{array}$ \\
\hline
\end{tabular}

Any COVID-19 includes a positive test result from PHE, or an inpatient diagnosis from UK Biobank inpatient hospital data or a death cause of COVID-19 infection Odds Ratio (95\%) were derived from logistic regression models, which were adjusted for birth year, sex, race/ethnicity, body mass index, smoking, alcohol use, Townsend deprivation index, annual household income, educational attainment, and Charlson Comorbidity Index (dementia excluded) 
Table 4 Individuals with any or specific neurodegenerative disease (exposed), compared to individuals without such a condition (unexposed) - an analysis among all participants with a positive test result for COVID-19

\begin{tabular}{|c|c|c|c|}
\hline & \multicolumn{2}{|l|}{ No.(\%) of cases } & \multirow[t]{2}{*}{ Odds Ratio ${ }^{a}$} \\
\hline & Exposed & Unexposed & \\
\hline \multicolumn{4}{|l|}{ COVID-19 related death } \\
\hline Any neurodegenerative disease & $195 / 718(27.2)$ & $986 / 17220(5.7)$ & $2.08(1.71,2.53)$ \\
\hline Primary neurodegenerative diseases & $132 / 512(25.8)$ & $1049 / 17426(6.0)$ & $1.91(1.52,2.40)$ \\
\hline Vascular neurodegenerative diseases & 47/143 (32.9) & $1134 / 17795(6.4)$ & $1.89(1.29,2.77)$ \\
\hline Other neurodegenerative diseases & $98 / 322(30.4)$ & $1083 / 17616(6.1)$ & $2.16(1.65,2.82)$ \\
\hline \multicolumn{4}{|c|}{ Selected specific neurodegenerative diseases } \\
\hline Alzheimer's disease & $54 / 220(24.5)$ & $1127 / 17718(6.4)$ & $1.58(1.12,2.21)$ \\
\hline Parkinson's disease & $52 / 196(26.5)$ & $1129 / 17742(6.4)$ & $1.97(1.39,2.78)$ \\
\hline
\end{tabular}

a Odds Ratio (95\%) were derived from logistic regression models, which were adjusted for birth year, sex, race/ethnicity, body mass index, smoking, alcohol use, Townsend deprivation index, annual household income, educational attainment, and Charlson Comorbidity Index

comorbidities, and did not differ greatly between primary neurodegenerative diseases and neurodegenerative diseases with a primary vascular component. The subgroup analysis among individuals with a positive test result for COVID-19 showed that, compared to other people, individuals with neurodegenerative disease had further a higher risk of death, after contracting COVID-19. Taken together, these results suggest that neurodegenerative disease is associated with an increased susceptibility to COVID-19, especially the severe form.

The association between neurodegenerative diseases and COVID-19 has been discussued in nine previous studies, with inconsistent findings [14-22]. Four cohort studies, conducted in the UK, Russia, and Italy, reported a positive association between dementia and COVID-19 hospitalization and death [14, 19-21]. Beside a shorter follow-up period and limited number of COVID-19 cases, these studies did not fully control for some important cofounders, such as socioeconomic and lifestyle factors. Similarly, a moderate increase in COVID-19 mortality (30, 95\%CI 13-49\%) was observed among PD patients in a US cohort study, without however adjusting for comorbidities [22]. Null association was reported in two studies of PD, both with relatively small sample size $[16,17]$. Using data from one single hospital in Wuhan, China, patients with AD were found to have a significantly shorter duration of hospital stay for COVID-19 [18]. To this end, the present study, based on a large community-based cohort and with careful consideration of selection bias and a wide range of potential confounders, demonstrated a clear association between various neurodegenerative diseases and a markedly increase risk of COVID-19, especially the severe and fatal form of COVID-19. This highlights the needs of special care or intervention, during the COVID-19 outbreak, for individuals with neurodegenerative diseases.

The underlying mechanisms for the observed association remain unclear. A possible explanation is the generally increased susceptibility to infection, possibly induced by dysregulated immune system inherently involved in the development of neurodegenerative diseases [28-30], including alterations in the peripheral immune responses $[31,32]$. In addition, chronic inflammation is commonly observed in neurodegenerative diseases and may also lead to increased risk of infection [30, 31]. We observed slightly higher $\mathrm{OR}$ for $\mathrm{AD}$ relative to $\mathrm{PD}$, which might imply special pathways linking AD with COVID-19, in addition to common mechanisms for the entire spectrum of neurodegenerative diseases. Based on experimental studies, SARS-CoV-2 binds to the target cell via transmembrane protein angiotensin-converting enzyme 2 (ACE2), which is expressed in various of organs [33]. Given that elevated ACE2 expression level has been observed in $\mathrm{AD}$ patients [34], it is possible that the excess in ACE2 in AD provides more cellular receptors when exposed to SARS-CoV-2, and therefore increases the vulnerability to COVID-19.

The major strength of our study is the use of a large community-based cohort, where data on exposure and outcome were collected prospectively and independently. The large sample size made it possible to perform detailed analysis for different subtypes of neurodegenerative disease, as well as for individuals with different characteristics. In addition, the rich information on sociodemographic and medical conditions enabled us to adjust for a wide range of confounders. Also, the application of a matched-cohort design in the sensitivity analysis demonstrated the robustness of our findings to age, as an 
important confounder. Finally, as multiple data sources were used for the identification of COVID-19 status, we were able to study COVID-19 with different levels of severity, any COVID-19 infection, COVID-19 requiring inpatient care, and COVID-19 related death.

There were several limitations in this study. First, under ascertainment of COVID-19 cases, especially cases with mild or no symptoms, is a concern of all population-based studies for COVID-19. Individuals with chronic diseases (e.g., neurodegenerative diseases) might have different access to health care and therefore different access to COVID-19 test. For example, patients undergo non-COVID-19 related health care might receive additional COVID-19 test. Thus number of potential under ascertainment cases of mild and no symptoms COVID-19 cases in exposed group and unexposed group is likely differential and would lead to selection bias in a study aiming to examine the association between neurodegenerative diseases and COVID-19. As a result, in the main analysis, we restricted the analyses to individuals who had received at least one COVID-19 test, instead of studying the entire UK Biobank population. While the tested population is strongly influenced by the testing policy, especially in the beginning of the pandemic due to the limited testing capacity, they were restricted in the inpatients with typical syndrome of respiratory system. To address this, we further performed a bias analysis, assuming 5 to $100 \%$ underestimate of COVID-19 outcomes among individuals without neurodegenerative diseases, whereas no underestimate among individuals with neurodegenerative diseases, and found reassuringly positive associations in even the relatively extreme scenarios. Second, we had no information on place of residence (e.g., care home) for the study participants, which might raise a concern that the noted positive association might have been influenced by the spreading of COVID-19 in care homes, assuming that more individuals with neurodegenerative diseases were residing in care homes than others. However, the outbreak in UK care homes mainly occurred in spring 2020 (i.e., March to early May) [27]. Together, based on data from Whole Care Home Testing Programme, including test results from all 9081 care homes for $65+$ in England, the positive rate of COVID-19 tests was as low as $3.9 \%$ (6747 out of 172,066$)$ between 11th May and 7th June 2020 [35]. We believe therefore that the spreading of COVID-19 in care homes did not pertain to the entire period of our study. In subgroup analyses by calendar periods, we set the time points according to the changing of test policy (April 27th 2020, July 7th 2020) and the dominant variants in the UK (December 17th 2020) [36]. We observed constantly increased risk of COVID-19 outcomes among individuals with neurodegenerative diseases before April 26th 2020, during April 27th 2020-July 6th 2020, during July 7th 2020 -December 16th 2020, and from December 17th 2020 onward. If the spreading of COVID-19 in care homes indeed contributed heavily to our results, we should have expected a specifically high OR during July 7th 2020 -December 16th 2020, as regular COVID19 tests have been offered in all care homes in the UK since July 7th 2020 [25]. We believe therefore that the spreading of COVID-19 in care homes was unlikely to explain completely our findings. This concern was further alleviated by the fact that we observed increased risk of COVID-19 related death in relation to neurodegenerative disease in the subgroup analysis of individuals tested positive for COVID-19. Third, somatic comorbidities were measured by diagnoses extracted from inpatient hospital data, as a result we might have missed milder comorbidities (e.g., diabetes) that did not require hospitalization. The adjustment of Charlson Comorbidity Index may not completely relieve the concern on confounding due to differential somatic conditions between individuals with neurodegenerative diseases and the unexposed group. Fourth, with only $5.5 \%$ response rate among the target population, the UK Biobank participants are not representative of the general population in the UK. Caution is therefore required when generalizing these findings to the whole UK or other populations [37]. Finally, as we defined neurodegenerative diseases according to inpatient hospital data, our findings might not be directly applicable to individuals with neurodegenerative diseases that are never attended by inpatient care.

\section{Conclusions}

Among the UK Biobank participants who underwent a test for SARS-CoV-2, a pre-existing diagnosis of neurodegenerative diseases was associated with a subsequently elevated risk of COVID-19, especially the severe form. Although validations from future studies are needed, these findings underscore the need for better surveillance and medical care for individuals with neurodegenerative diseases during the COVID-19 outbreak, both before and after contracting the infection.

\section{Abbreviations}

ACE2: Angiotensin-converting enzyme 2; AD: Alzheimer's disease; BMI: Body mass index; COVID-19: Coronavirus disease 2019; CSE: Certificate of Secondary Education; HES: Hospital Episode Statistics; ICD: International Classification of Diseases codes; NHS: National Health Service; NVQ: National Vocation Qualifications; ORs: Odd ratios; PD: Parkinson's disease; PEDW: Patient Episode Database for Wales; PHE: Public Health England; SARS-CoV-2: Severe acute respiratory syndrome coronavirus 2; UK: United Kingdom; WHO: World Health Organization. 


\section{Supplementary Information}

The online version contains supplementary material available at https://doi. org/10.1186/s12883-021-02536-7.

Additional file 1 Supplementary Fig. 1. Risk of COVID-19 among individuals with any or specific neurodegenerative disease, compared to matched individuals without such a condition. Supplementary Fig. 2 Changes of odds ratios with $95 \%$ confidence intervals ${ }^{\text {a }}$, by assuming $5-100 \%$ underestimation of the studied outcomes among individuals without neurodegenerative diseases. Supplementary Table 1 A Summary of previous studies addressing the association between neurodegenerative diseases and COVID-19. Supplementary Table 2. International Classification of Disease (ICD) codes, ninth (ICD-9) and tenth (ICD-10) revisions for diagnoses used in this study.

\section{Acknowledgements}

We thank the team members and colleagues involved in West China Biomedical Big Data Center- UK Biobank project for their support.

\section{Authors' contributions}

$H S$, FF, and $Y H H$ were responsible for the study concept and design. $Z Y, Y H$, $Y S$, and $Y Q$ did the data and project management. $\mathrm{HY}, \mathrm{CH}, \mathrm{WC}$ and $\mathrm{HZ}$ did the data cleaning and analysis. YHH, HY, MF, UV, HS, and FF interpreted the data. $\mathrm{YHH}, \mathrm{HY}, \mathrm{HS}$, and $\mathrm{FF}$ drafted the manuscript, $\mathrm{YHH}, \mathrm{MF}, \mathrm{UV}, \mathrm{HS}$, and FF revised the manuscript. All authors approved the final manuscript as submitted and agree to be accountable for all aspects of the work.

\section{Funding}

This work is supported by the National Natural Science Foundation of China (No. 81971262 to HS), West China Hospital COVID-19 Epidemic Science and Technology Project (No. HX-2019-nCoV-014 to HS), Sichuan University Emergency Grant (No. 2020scunCoVyingji10002 to HS), National Clinical Research Center for Geriatrics, West China Hospital, Sichuan University (No. Z20201013 to HS), Project Grant form Science \& Technology Department of Sichuan Providence (2020YFS0575 to HS), and NordForsk grant (105668 to UV and FF). The funding agencies did not have any role in the design of study, data collection, data analysis, interpretation or writing the manuscript.

\section{Availability of data and materials}

Data from the UK Biobank (http://www.ukbiobank.ac.uk/) are available to all researchers upon making an application. Part of this research was conducted using the UK Biobank Resource under Application 54803.

\section{Declarations}

Ethics approval and consent to participate

The UK Biobank has full ethical approval from the NHS National Research Ethics Service (reference number: 16/NW/0274). All participants of UK Biobank provided written consent for participation. This study was also approved by the biomedical research ethics committee of West China Hospital (reference number: 2020.661).

\section{Consent for publication}

Not applicable.

\section{Competing interests}

We declare no competing interests.

\footnotetext{
Author details

'West China Biomedical Big Data Center and National Clinical Research Center for Geriatrics, West China Hospital, Sichuan University, Guo Xue Lane 37, Chengdu 610041, China. ${ }^{2}$ Medical Big Data Center, Sichuan University, Chengdu 610041, China. ${ }^{3}$ Institute of Environmental Medicine, Karolinska Institutet, 17177 Stockholm, Sweden. ${ }^{4}$ Division of Nephrology, Kidney Research Institute, State Key Laboratory of Biotherapy and Cancer Center, West China Hospital, Sichuan University, Chengdu 610041, China. ${ }^{5}$ Center of Public Health Sciences, Faculty of Medicine, University of Iceland, 101 Reykjavík, Iceland.
}

${ }^{6}$ Department of Medical Epidemiology and Biostatistics, Karolinska Institutet, 17177 Stockholm, Sweden.

Received: 11 October 2021 Accepted: 26 December 2021

Published online: 07 January 2022

\section{References}

1. World Health Organization (WHO). Director-General's opening remarks at the media briefing on COVID-19-11 March 2020. 2020;11. https://www. who.int/director-general/speeches/detail/who-director-general-s-openi ng-remarks-at-the-media-briefing-on-covid-19\%2D\%2D-20-march-2020. accessed 11 Dec 2020.

2. Wu D, Wu T, Liu Q, Yang Z. The SARS-CoV-2 outbreak: what we know. Int J Infect Dis. 2020;94:44-8. https://doi.org/10.1016/j.jij. 2020.03.004.

3. Onder G, Rezza G, Brusaferro S. Case-fatality rate and characteristics of patients dying in relation to COVID-19 in Italy. JAMA. 2020;323(18):17756. https://doi.org/10.1001/jama.2020.4683.

4. Wu JT, Leung K, Bushman M, et al. Addendum: estimating clinical severity of COVID-19 from the transmission dynamics in Wuhan. China Nat Med. 2020;26(7):1149-50. https://doi.org/10.1038/s41591-020-0920-6.

5. Guan W-J, Ni Z-Y, HuY, et al. Clinical characteristics of coronavirus disease 2019 in China. N Engl J Med. 2020;382(18):1708-20. https://doi.org/10. 1056/nejmoa2002032.

6. Zhou F, Yu T, Du R, et al. Clinical course and risk factors for mortality of adult inpatients with COVID-19 in Wuhan, China: a retrospective cohort study. Lancet. 2020;395(10229):1054-62. https://doi.org/10.1016/s01406736(20)30566-3.

7. Burns A, lliffe S. Alzheimer's disease. BMJ. 2009;338:b158. https://doi.org/ 10.1136/bmj.b158.

8. Prince M, Bryce R, Albanese E, Wimo A, Ribeiro W, Ferri CP. The global prevalence of dementia: a systematic review and metaanalysis. Alzheimers Dement. 2013;9(1):63-75 e2. https://doi.org/10.1016/j.jalz.2012.11. 007.

9. Kalia LV, Lang AE. Parkinson's disease. Lancet. 2015;386(9996):896-912. https://doi.org/10.1016/j.jalz.2012.11.007.

10. Cuffaro L, Di Lorenzo F, Bonavita S, Tedeschi G, Leocani L, Lavorgna L. Dementia care and COVID-19 pandemic: a necessary digital revolution. Neurol Sci. 2020;41(8):1977-9. https://doi.org/10.1007/ s10072-020-04512-4.

11. Victorino DB, Guimarães-Marques M, Nejm M, Scorza FA, Scorza CA. COVID-19 and Parkinson's disease: are we dealing with short-term impacts or something worse? J Parkinsons Dis. 2020;10(3):899-902. https://doi.org/10.3233/jpd-202073.

12. Wang H, LiT, Barbarino P, et al. Dementia care during COVID-19. Lancet. 2020;395(10231):1190-1. https://doi.org/10.1016/s0140-6736(20)30755-8.

13. Prasad S, Holla WV, Neeraja K, et al. Parkinson's disease and COVID-19: perceptions and implications in patients and caregivers. Mov Disord. 2020;35(6):912-4. https://doi.org/10.1002/mds.28088.

14. Atkins JL, Masoli JAH, Delgado J, et al. Preexisting comorbidities predicting COVID-19 and mortality in the UK biobank community cohort. J Gerontol A Biol Sci Med Sci. 2020;75(11):2224-30. https://doi.org/10. 1093/gerona/glaa183.

15. Bianchetti A, Rozzini R, Guerini F, et al. Clinical presentation of COVID19 in dementia patients. J Nutr Health Aging. 2020;24(6):560-2. https://doi.org/ 10.1007/s12603-020-1389-1.

16. Fasano A, Cereda E, Barichella M, et al. COVID-19 in Parkinson's disease patients living in Lombardy. Italy Mov Disord. 2020;35(7):1089-93. https:// doi.org/10.1002/mds.28176.

17. Hwang JM, Kim JH, Park JS, Chang MC, Park D. Neurological diseases as mortality predictive factors for patients with COVID-19: a retrospective cohort study. Neurol Sci. 2020;41(9):2317-24. https://doi.org/10.1007/ s10072-020-04541-z.

18. Li J, Long $X$, Huang H, et al. Resilience of Alzheimer's disease to COVID-19. J Alzheimers Dis. 2020;77(1):67-73. https://doi.org/10.3233/jad-200649.

19. Giorgi Rossi P, Marino M, Formisano D, Venturelli F, Vicentini M, Grilli R. Characteristics and outcomes of a cohort of COVID-19 patients in the province of Reggio Emilia. Italy PloS one. 2020;15(8):e0238281. https:// doi.org/10.1371/journal.pone.0238281. 
20. Docherty AB, Harrison EM, Green CA, et al. Features of 20133 UK patients in hospital with covid-19 using the ISARIC WHO clinical characterisation protocol: prospective observational cohort study. BMJ. 2020;369:m1985. https://doi.org/10.1136/bmj.m1985.

21. Munblit D, Nekliudov NA, Bugaeva P, et al. StopCOVID cohort: An observational study of 3,480 patients admitted to the Sechenov University hospital network in Moscow city for suspected COVID-19 infection. Clin Infect Dis. 2020:ciaa1535. https://doi.org/10.1093/cid/ciaa1535.

22. Zhang Q, Schultz JL, Aldridge GM, Simmering JE, Narayanan NS. Coronavirus disease 2019 case fatality and Parkinson's disease. Mov Disord. 2020;35(11):1914-5. https://doi.org/10.1002/mds.28325.

23. Bycroft C, Freeman C, Petkova D, et al. The UK biobank resource with deep phenotyping and genomic data. Nature. 2018;562(7726):203-9. https://doi.org/10.1038/s41586-018-0579-z.

24. UK Biobank COVID-19 test results data 2020 https://biobank.ndph.ox.ac uk/showcase/exinfo.cgi?src=COVID19_tests. accessed 11 Dec 2020.

25. Regular retesting rolled out for care home staff and residents. https:// www.gov.uk/government/news/regular-retesting-rolled-out-for-carehome-staff-and-residents. accessed March 29, 2021.

26. Townsend P, Phillimore P, Beattie A. Health and deprivation: inequality and the north: Routledge; 1988.

27. COVID19_Care_Homes_22_July.Pdf. https://assets.publishing.service.gov uk/government/uploads/system/uploads/attachment_data/file/903085/ COVID19_Care_Homes_22_July.pdf. accessed 29 Mar 2021.

28. Cunningham C, Wilcockson DC, Campion S, Lunnon K, Perry VH. Central and systemic endotoxin challenges exacerbate the local inflammatory response and increase neuronal death during chronic neurodegeneration. J Neurosci. 2005;25(40):9275-84. https://doi.org/10.1523/jneurosci. 2614-05.2005.

29. Cao W, Zheng H. Peripheral immune system in aging and Alzheimer's disease. Mol Neurodegener. 2018;13(1):51. https://doi.org/10.1186/ s13024-018-0284-2.

30. Akbar AN, Gilroy DW. Aging immunity may exacerbate COVID-19. Science. 2020;369(6501):256-7. https://doi.org/10.1126/science.abb0762.

31. Lucin KM, Wyss-Coray T. Immune activation in brain aging and neurodegeneration: too much or too little? Neuron. 2009;64(1):110-22. https:// doi.org/10.1016/j.neuron.2009.08.039.

32. Dilger RN, Johnson RW. Aging, microglial cell priming, and the discordant central inflammatory response to signals from the peripheral immune system. J Leukoc Biol. 2008;84(4):932-9. https://doi.org/10.1 189/jlb.02081 08.

33. Ni W, Yang $X$, Yang $D$, et al. Role of angiotensin-converting enzyme 2 (ACE2) in COVID-19. Crit Care. 2020;24(1):422. https://doi.org/10.1186/ s13054-020-03120-0.

34. Lim KH, Yang S, Kim SH, Joo JY. Elevation of ACE2 as a SARS-CoV-2 entry receptor gene expression in Alzheimer's disease. J Inf Secur. 2020;81(3):e33-e4. https://doi.org/10.1016/j.jinf.2020.06.072.

35. GOV.UK. "Vivaldi 1: COVID-19 Care Homes Study Report" https://www. gov.uk/government/publications/vivaldi-1-coronavirus-covid-19care-homes-studyreport/vivaldi-1-covid-19-care-homes-study-report . accessed Mar 27, 2021.

36. GOV.UK. COVID-19 variants: genomically confirmed case numbers https://www.gov.uk/government/publications/covid-19-variants-genom ically-confirmed-case-numbers. accessed 27 Mar 2021.

37. Fry A, Littlejohns TJ, Sudlow C, et al. Comparison of sociodemographic and health-related characteristics of UK biobank participants with those of the general population. Am J Epidemiol. 2017;186(9):1026-34. https:// doi.org/10.1093/aje/kw×246.

\section{Publisher's Note}

Springer Nature remains neutral with regard to jurisdictional claims in published maps and institutional affiliations.

Ready to submit your research? Choose BMC and benefit from:

- fast, convenient online submission

- thorough peer review by experienced researchers in your field

- rapid publication on acceptance

- support for research data, including large and complex data types

- gold Open Access which fosters wider collaboration and increased citations

- maximum visibility for your research: over 100M website views per year

At BMC, research is always in progress.

Learn more biomedcentral.com/submissions 